\title{
Immunoglobulin G4-Related Orbital Disease with Bilateral Optic Perineuritis and Maxillary Nerves Involvement: A Case Report
}

\author{
Chih-Heng Hung (D) C Cheng-Yu Lo
}

Received: August 5, 2020 / Accepted: October 3, 2020 / Published online: October 17, 2020

(C) The Author(s) 2020

\begin{abstract}
Immunoglobulin G4-related optic neuropathy caused by optic perineuritis is a rare complication of immunoglobulin G4-related disease (IgG4-RD). Herein, we report a 38-year-old Asian man with history of sinusitis who presented with painless blurred vision and proptosis for over 6 months. Examination with the Hertel exophthalmometer revealed $21.5 \mathrm{~mm}$ on both eyes. Magnetic resonance imaging revealed a doughnut sign encircling the right optic nerve, bilateral tram-track signs on both optic nerves, enlarged bilateral maxillary nerves with perineural spreading to the infraorbital nerves, hypertrophy of extraocular muscles, and pansinusitis. Visual evoked potentials displayed bilateral delayed P100 latency, indicating bilateral optic neuropathy. Biopsy with functional
\end{abstract}

C.-H. Hung ( $₫)$

Department of Ophthalmology, Cathay General

Hospital, Taipei, Taiwan

e-mail: peteroph@hotmail.com

C.-Y. Lo

Department of Pathology, Cathay General Hospital,

Taipei, Taiwan endoscopic sinus surgery demonstrated diffuse dense lymphoplasmacytic infiltrate and fibrosis. IgG4-positive plasma cells exceeded 50 cells per high-power field while the overall IgG4/IgG ratio was above $40 \%$. Serological studies unveiled extremely high serum concentrations of IgG4 (2650 mg/dL), and the calculated serum IgG4/IgG ratio was $100 \%$. These comprehensive features supported the diagnosis of IgG4-RD with bilateral optic perineuritis, branches of trigeminal nerve involvement, and pansinusitis. The visual acuity improved slightly following the initiation of treatment with corticosteroids, but it became worse again during the tapering course. Following another course of corticosteroids followed by subsequent immunosuppressant treatment with azathioprine, vision in both eyes ultimately improved during the 2-year follow-up period.

Keywords: IgG4-related disease; Maxillary nerve; Optic perineuritis; Orbital disease 


\section{Key Summary Points}

IgG4-related optic neuropathy (IgG4$\mathrm{RON}$ ) caused by optic perineuritis is a rare complication of IgG4-related disease (IgG4-RD).

Limited information is available in the literature on the clinical and radiologic risk factors associated with the optic perineuritis type of IgG4-RON.

We report a rare case of IgG4-RD with bilateral optic perineuritis, enlarged maxillary nerves with perineural spreading to infraorbital nerves, and pansinusitis; with the diagnosis confirmed by histopathologic study of sinus biopsy specimens.

Magnetic resonance imaging is a useful tool for diagnosing cranial nerve perineuritis, with the presence of the "doughnut" sign and "tram-track" sign indicative of inflamed soft tissues surrounding the cranial nerve.

The case described here is a "pure" case of optic perineuritis secondary to IgG4-RD, and a successful post-treatment visual outcome was obtained with treatment with oral corticosteroids followed by azathioprine.

\section{DIGITAL FEATURES}

This article is published with digital features, including a summary slide, to facilitate understanding of the article. To view digital features for this article go to https://doi.org/10.6084/ m9.figshare.13027988.

\section{INTRODUCTION}

Immunoglobulin G4-related disease (IgG4-RD) is an immune-mediated fibroinflammatory disease characterized by tumefactive lesion with abundant IgG4-positive plasma cells infiltrating into multiple organs. IgG4-RD was first described in the pancreas as a cause of "lymphoplasmacytic sclerosing pancreatitis." Other frequent sites of involvement include the thyroid gland, aorta, liver, bile ducts, retroperitoneum, and lymph nodes. In the head and neck lesions have been identified in the meninges, pituitary gland, salivary glands, middle ear, and sinuses.

IgG4-related ophthalmic disease (IgG4-ROD) is present in $23 \%$ of patients with IgG4-RD, in whom the lacrimal gland is the most commonly involved structure, followed by orbital fat and extraocular muscles, albeit optic neuropathy is rare [1]. The presentation of patients with IgG4ROD is variable and generally manifests as organ dysfunction, pain, or swelling, such as lacrimal gland swelling or exophthalmos from orbital soft tissue swelling. In two studies, IgG4related optic neuropathy (IgG4-RON) presented in $5-9 \%$ of the patients in two multicenter cohorts with IgG4-ROD. Among 12 cases of IgG4-RON, ten were of the compressive type and the other two were of the infiltrative type $[2,3]$. Poor vision and prolonged duration of symptoms at initial presentation resulted in poor post-treatment visual outcome [2].

However, only limited information is available in the literature on the clinical and radiologic risk factors associated with the optic perineuritis type of IgG4-RON. There are very few reported cases in which secondary optic perineuritis caused by IgG4-RD has been described. Herein, we report a case of IgG4-RD with bilateral optic perineuritis and branches of trigeminal nerves involvement. The successful post-treatment visual outcome was obtained through early treatment with oral corticosteroids followed by azathioprine.

The patient provided informed consent for his clinical details to be known and signed an informed consent form for publication of the case. This study was conducted in compliance with the guidelines of the Declaration of Helsinki. 


\section{CASE PRESENTATION}

A 38-year-old Asian man with a history of sinusitis status presented at the Ophthalmology outpatient department following a surgical intervention at another hospital 3 months previously, with complaints of gradual painless blurred vision and proptosis for 6 months. There was no periocular sensory change or recent trauma. His family history was unremarkable.

On ophthalmic examination, best-corrected visual acuity (BCVA) was 20/30 on the right eye (OD) and 20/40 on the left eye (OS). Slit-lamp and funduscopic examination results were normal. The pupil reacted sluggishly without a relative afferent pupillary defect. Color vision was normal. Ocular motility was intact without diplopia, but exophthalmometry measurements were $21.5 \mathrm{~mm}$ with base $112 \mathrm{~mm}$ using one accurately calibrated Inami exophthalmometer (Hertel K-0161; Inami \& Co., Ltd., Bunkyo-ku, Tokyo, Japan) on both eyes (OU). There was neither periocular sensory change nor palpated mass in the lower eyelid.

Thyroid function tests revealed normal free thyroxine and thyroid-stimulating hormone levels, but serological studies showed extremely high serum concentrations of IgG4 $(2650 \mathrm{mg} /$ $\mathrm{dL}$; normal range $<135 \mathrm{mg} / \mathrm{dL}$ ); elevated serum concentrations of $\operatorname{IgG}(2630 \mathrm{md} / \mathrm{dL}$; normal range $751.0-1560.0 \mathrm{mg} / \mathrm{dL}$ ); abnormal erythrocyte sedimentation rate $(31 \mathrm{~mm} / \mathrm{h}$; normal range $<15 \mathrm{~mm} / 1 \mathrm{~h}$ ); and high levels of total IgE (1903.00 IU/dL; normal range $<87.00 \mathrm{IU} / \mathrm{mL}$ ). The calculated serum IgG4/ IgG ratio was $100 \%$ (normal range $<6 \%$ ). No eosinophilia was found in the blood.

Postcontrast T1-weighted fat-saturated magnetic resonance imaging (MRI) images showed a "doughnut" sign encircling the right optic nerve, and inferior sheath enhancement surrounding the left optic nerve was visible on the coronal MRI images (Fig. 1a). Bilateral "tramtrack" signs were evident on the axial images (Fig. 1b). Bilateral enhancing lesions at the pterygoid plate, pterygomaxillary fissure, and sella region, with involvement of both maxillary nerves, were visualized using gadolinium- enhanced T1-weighted MRI (Fig. 1c, d). Bilateral infraorbital nerve involvement was also evident on the MR images (Fig. 1d, e). Enlargement of extraocular muscles, especially severe at the bilateral inferior rectus, right medial rectus, bilateral superior rectus, and bilateral lateral rectus muscles, was noted on coronal MRI images (Fig. 1e). Mucosal thickening in the ethmoid, maxillary, sphenoid, and frontal sinuses was also visible.

We referred this patient to the Department of Otolaryngology in our hospital for surgical biopsy. Biopsy with functional endoscopic sinus surgery was performed, and a tissue specimen measuring $0.5 \times 0.3 \times 0.2 \mathrm{~cm}$ was sent to the Department of Pathology for further testing. The result revealed chronic sinusitis with dense infiltrate of lymphocytes and plasma cells. Chronic inflamed granulation tissue, aggregates of foamy histiocytes, and fibrosis of sinonasal tissue were also noted (Fig. 2a-c). Organisms were not identified in the Periodic Acid-Schiff stain and the Acid-fact stain tests. Immunhistochemistry revealed CD138 accentuated plasma cells (Fig. 2d) and highlighted numerous IgG4-positive plasma cells (> 50 IgG4-positive plasma cells per high-power field), of which over $40 \%$ were IgG-positive plasma cells (Fig. 2e, f).

The patient underwent a treatment regimen comprising oral systemic corticosteroids (prednisolone $1 \mathrm{mg}$ per $\mathrm{kg}$ body weight daily) for 2 weeks followed by slow tapering. Visual acuity improved to $20 / 25$ (OU) 1 month after initiating treatment. Four months after the initial treatment, the vision got worse again, with the BCVA dropping to 20/30 (OD) and 20/50 (OS), respectively. Visual field test showed parafoveal scotoma (OD) and an enlarged blind spot (OS). Visual evoked potentials indicated bilateral delayed P100 latency (Fig. 1f). No active lesions were found after systemic screening. Based on the combined clinical, radiographic, and histopathologic findings, the final diagnosis was IgG4-RD with bilateral optic perineuritis, branches of trigeminal nerves involvement, and pansinusitis.

The patient underwent another course of oral prednisolone daily for 2 weeks with gradually tapering of dosage. This was followed by 


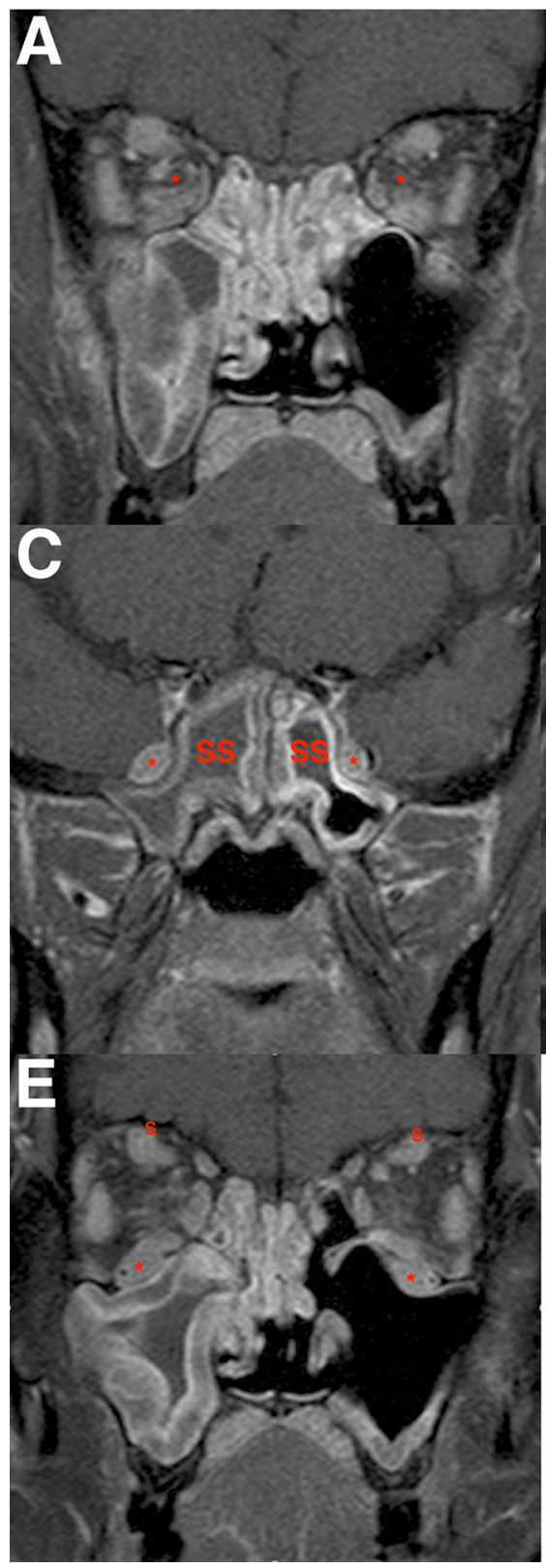

Fig. 1 Magnetic resonance imaging (MRI) images of a 38-year-old Asian man who presented with bilateral optic perineuritis and maxillary nerve and infraorbital nerve involvement. Pansinusitis was also found. a Coronal MRI revealed a doughnut sign encircling the right optic nerve (asterisk) and inferior sheath enhancement surrounding the left optic nerve (asterisk). Mucosal thickening in the ethmoid and maxillary sinuses was evident. b Axial MRI illustrated bilateral tram-track signs. The perineuritis was especially posterior located. c Coronal MRI highlighted bilateral maxillary nerves (asterisk). Mucosal thickening in

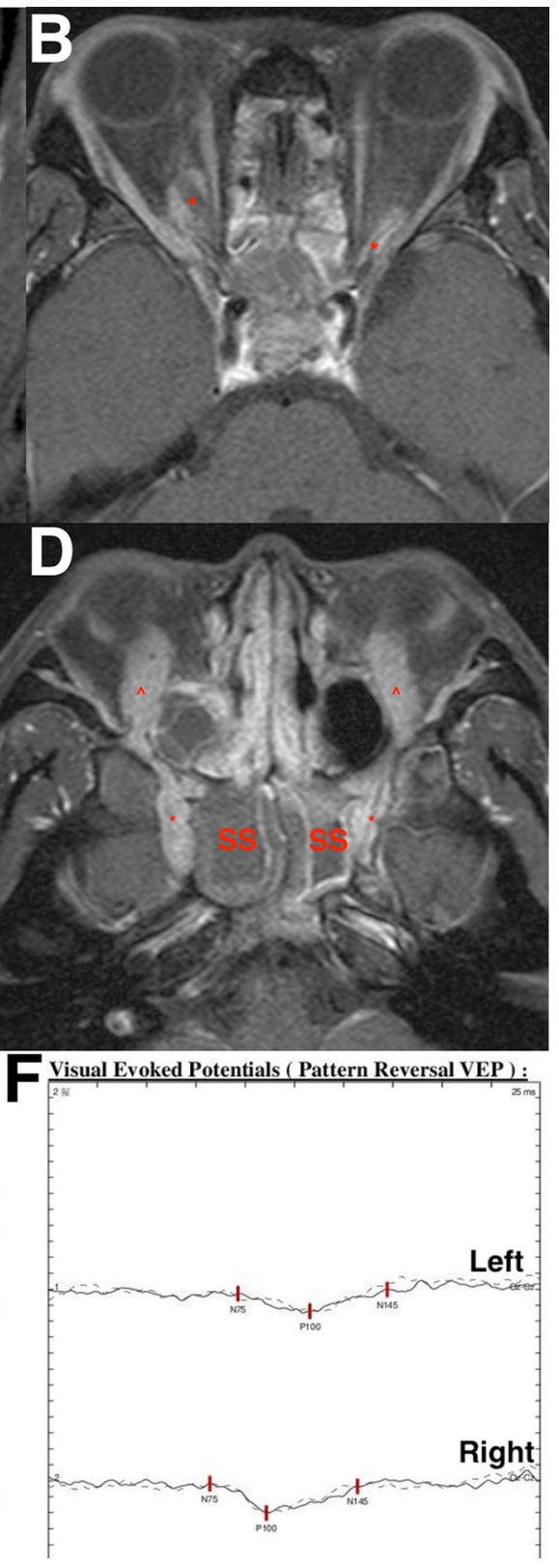

the sphenoid sinus (SS) was disclosed. d Axial MRI displayed both maxillary nerves (asterisk) and infraorbital nerves (arrowhead). Mucosal thickening in the SS was disclosed. e Coronal MRI revealed typical infraorbital nerve (asterisk) involvement. The supraorbital nerve $(S)$ was enlarged. Enlargement of extraocular muscles, especially severe at the bilateral inferior rectus, right medial rectus, bilateral superior rectus, and bilateral lateral rectus muscles, was noted. f Visual evoked potentials indicated bilateral delayed P100 latency 


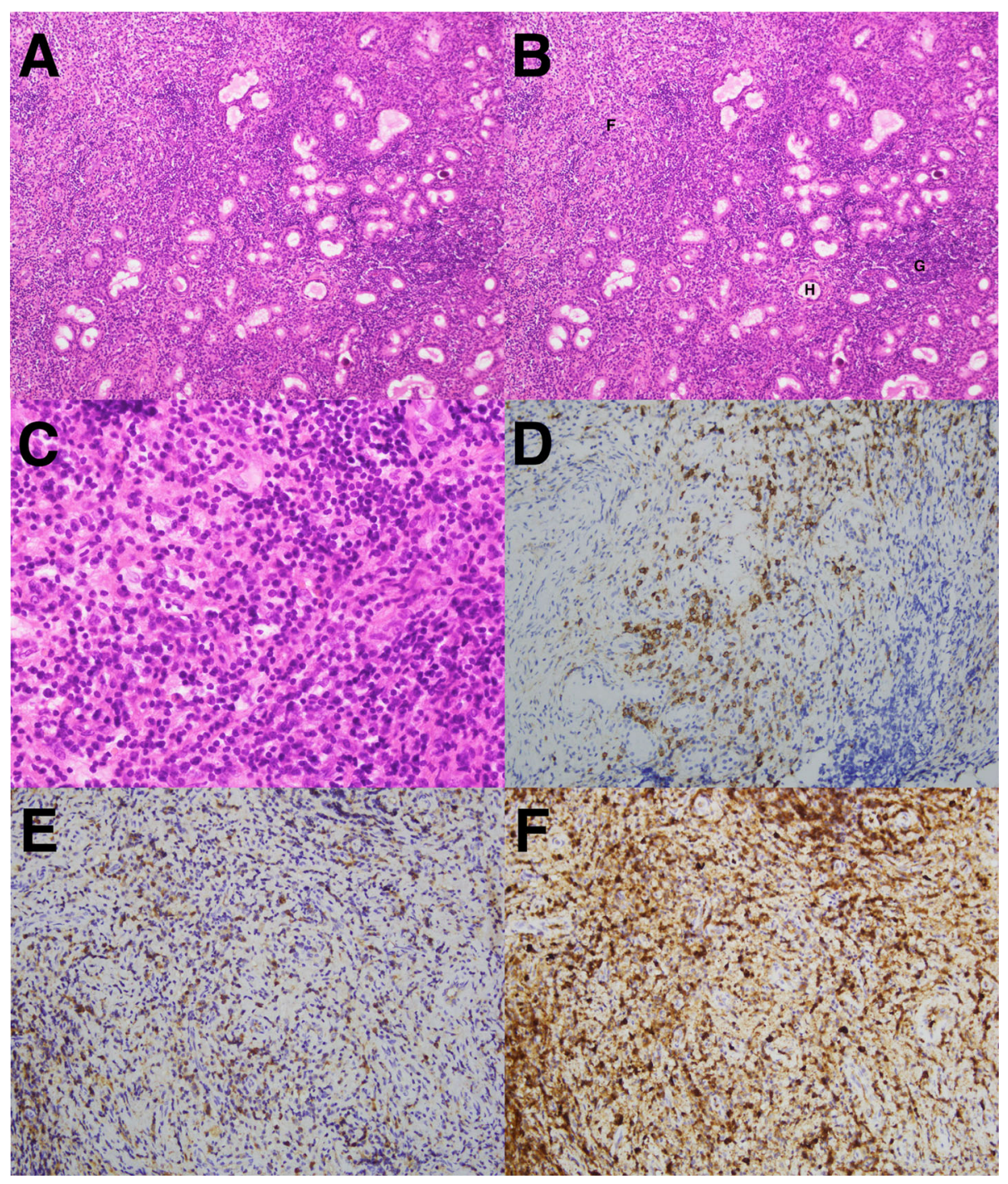

Fig. 2 a, b Histopathology showed a picture of chronic sinusitis with dense infiltrate of lymphocytes and plasma cells. Chronic inflamed granulation $(G)$, aggregates of foamy histiocytes $(H)$, and fibrosis of sinonasal tissue $(F)$ were noted $(\times 100)$. c Abundant lymphocytes and plasma cells $(\times 400)$. d Immunohistochemistry with CD138 revealed plasma cells $(\times 100)$.

treatment with the immunosuppressive agent azathioprine (50 mg, twice daily). The final BCVA was 20/20 (OD) and 20/25 (OS) during the follow-up period of 2 years. e Immunohistochemistry marked immunoglobulin G $(\mathrm{IgG})$-positive plasma cells $(\times 100)$. f Immunohistochemistry revealed numerous IgG4-positive plasma cells $(\times 100)$. The ratio of IgG4-positive cells to IgG-positive cells was $>40 \%$

\section{DISCUSSION}

The diagnosis of IgG4-RD is often challenging, requiring careful correlation of pathologic and radiologic findings with the patient's clinical features. Biopsy remains the cornerstone of diagnosing in IgG4-RD [4-6]. However, biopsy of the optic nerves involves high risks, 


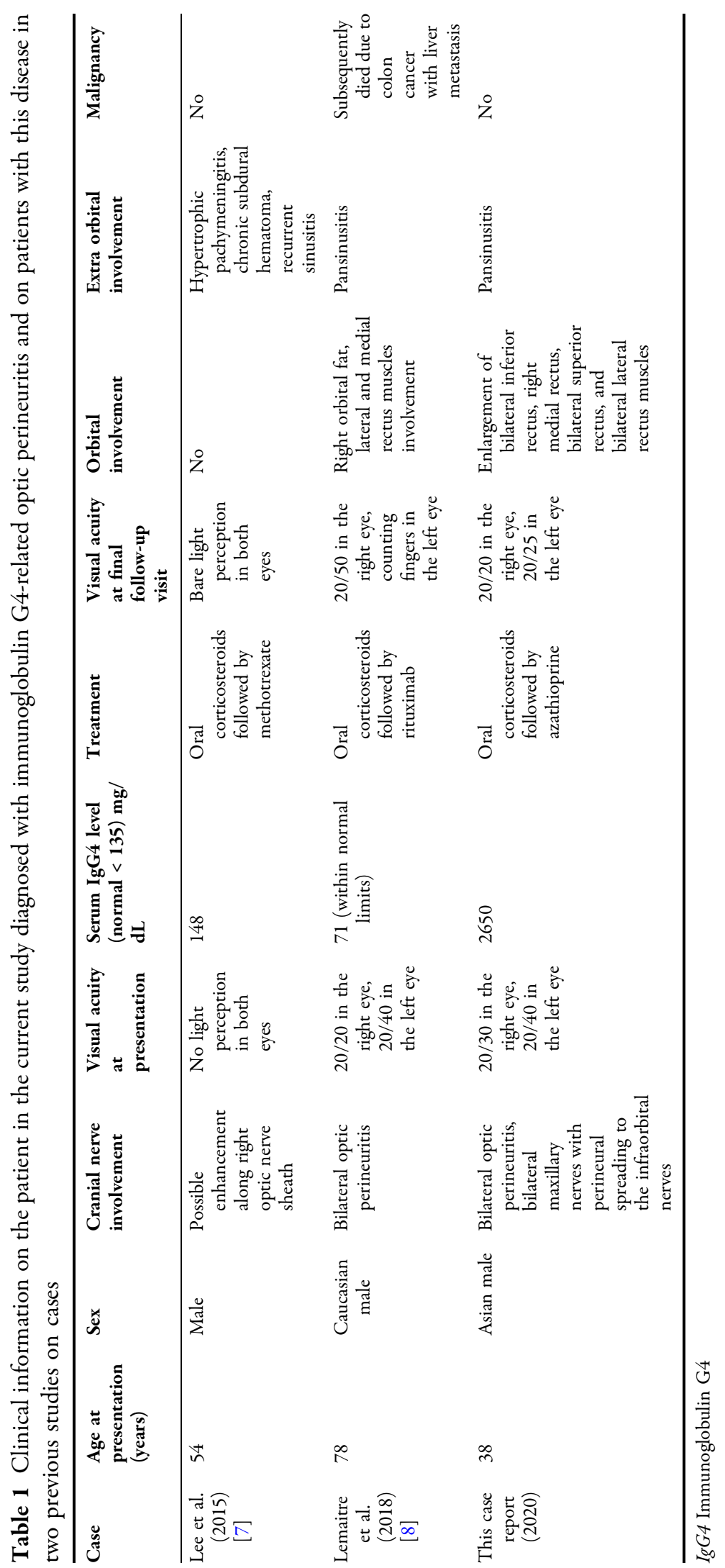


especially with respect to damage the optic nerve, which could result in blindness. To our best knowledge, there are only two reported cases of biopsy-proved IgG4-related optic perineuritis. In one case of idiopathic hypertrophic pachymeningitis, optic perineuritis, and sinusitis, the patient presented with no light perception in both eyes; a dural biopsy was performed to confirm the diagnosis of IgG4-RD [7]. In the second case, the patient presented with optic perineuritis, abdominal mass, and sinusitis; a final mesenterial biopsy was performed to confirm this probable case of IgG4RD [8]. It should be noted that up to $44 \%$ of patients with IgG4-RD have been reported to suffer from allergic diseases, such as asthma or sinusitis [9]. Tiegs-Heiden et al. reported that among the patients with IgG4-ROD in their study, sinusitis was present in 89\% [10]. Eosinophilia associated with recurrent sinusitis or asthma has been found to be a prominent feature in patients with definite IgG4-RD [11]. The inflammatory mucosal thickening noted in the paranasal sinuses has an unknown association with IgG4-ROD, with a potential link between IgG4 positivity and Th2 immune response being one possibility presented [11]. The presence of sinusitis may offer an additional clue when the diagnosis of IgG4-RD is considered. In the present case of IgG4-RD, the patient presented with bilateral optic perineuritis, branches of trigeminal nerves involvement, and pansinusitis. The final diagnosis was confirmed by immunochemistry tests, revealing IgG4positive plasma cells infiltrating the sinus tissue. Thus, sinus biopsy may be the easiest approach to obtain surgical specimens for testing and, consequently, a definite diagnosis in cases combined with optic nerve involvement. The clinical courses of prior cases with IgG4-related optic perineuritis are summarized in Table 1 $[7,8]$.

In the published case reports, patients with idiopathic optic perineuritis usually presented with ocular pain, pain with eye movement, and decreased vision, all symptoms which represent a form of idiopathic orbital inflammatory disease in which the specific target tissue is the optic nerve sheath and not the axons of the optic nerve [12]. Most patients with idiopathic optic perineuritis have reported a good recovery of visual acuity. Regarding those with poor recovery, the related factors were delay in initiating treatment and presence of progressive disease or additional ocular pathology [12]. However, persons can develop optic perineuritis secondary to an identifiable systemic disorder, such as syphilis, Behçet's disease, Crohn's disease, sarcoidosis, and granulomatosis with polyangiitis. In addition, IgG4-RD has recently been recognized as an inflamed cause of orbital inflammatory disease and can involve the optic nerve, although there are no reports to date of it causing a "pure" optic perineuritis in the orbit, with the exception of two reported cases, one of pachymeningitis that also involved the optic nerve sheath and the other of the serum IgG4 level not meeting the criteria $[7,8,12]$.

Optic neuropathy can be affected by IgG4RD through two mechanisms. First, the nerves become compressed by the mass effect of local IgG4-RD involvement of non-neural tissues. Second, the epineurium might be involved with chronic inflammatory aggregates caused by IgG4-RD, with or without further infiltration into the perineurium [13]. The prognosis of vision in patients with IgG4-RON has been diverse, complicated by the fact that information on the optic perineuritis type of IgG4-RON is extremely limited. In Lai et al.'s cohort study, three of six patients (50\%) with IgG4-RON who presented with poor vision had irreversible, permanent visual loss (BCVA worse than 20/200) [2]. Only two clinical cases on the optic perineuritis type of IgG4-RON have been reported to date. In one patient, the final state of vision was only the perception of bare light in both eyes, and the other patient suffered a loss of visual acuity down to 20/50 (OD) and counting fingers (OS) after corticosteroid treatment followed by rituximab treatment (this patient died 2 years later due to colon adenocarcinoma with multiple metastasis) [7, 8]. Three of the four eyes (75\%) of these two patients suffered permanent visual loss due to the IgG4-related optic perineuritis [7, 8]. A poor prognosis seems to be more likely in this subtype of IgG4-RON compared with the cases in Lai et al.'s cohort study [2]. However, in our case, the final BCVA remained good through 
treatment with oral corticosteroids followed by azathioprine. One possible explanation for the difference in outcome between these cases might be the state of vision at presentation and the duration of symptoms. Poor vision and prolonged duration of symptoms at initial presentation have been reported to result in poor post-treatment visual outcome $[2,12]$.

Data on the treatment of IgG4-RON are sparse, with only small case series and anecdotal reported experiences. The authors of one study reported that corticosteroids worked well for the compressive type of IgG4-RON but that disease relapsed after tapering medication [2]. Such high recurrence rates highlight the need for a corticosteroid-sparing treatment in most cases of IgG-RON. Regarding the optic perineuritis type of IgG4-RON, treatment with initial corticosteroids alone might prove beneficial, but relapses on a tapering dose of corticosteroids have been reported [7, 8]. Treatments with intravenous methylprednisolone or oral prednisolone for optic perineuritis or IgG4-RON have also been reported by individual practitioners $[2,7,8,12]$. In the present case, the disease initially responded well to treatment with corticosteroids, but the patient's vision became worse during the tapering course. After subsequent treatment with the immunosuppressanth azathioprine, the patient's vision ultimately did improve. Conventional immunosuppressive agents (such as azathioprine, methotrexate, cyclophosphamide, and mycophenolate mofetil) might remain effective in cases of IgG4-RD. B-cell depletion induced by rituximab has shown a good response in inducing remission in steroid-resistant disease, although the disease recurred in some cases $[14,15]$. Radiotherapy could exceptionally be employed in refractory cases but radiation optic neuropathy should be taken into consideration $[15,16]$. The present case and the two previous cases all showed refractory treatment history, indicating the need for a long-term treatment of optic perineuritis [7, 8]. Informing the patient with IgG4-RON of the possible corticosteroidsparing maintenance therapy may be necessary. More cases should be collected for further evaluation of the long-term outcome.
Involvement of the bilateral infraorbital nerve is relatively common and pathognomonic in cases of IgG-ROD. Two studies have reported that the infraorbital nerve was enlarged in approximately $30-38.5 \%$ of their IgG4-ROD cases $[3,10]$. The frontal nerves, supraorbital nerves, infraorbital nerves, lesser palatine nerves, and superior alveolar nerves were the involved sites of the trigeminal nerves in these patients $[3,17]$. Lesion of the trigeminal nerve branch was particularly unique, and $64 \%$ of trigeminal nerve branch lesions observed in Sogabe et al.'s study were bilateral [3]. Previous histopathologic assessment has revealed that IgG4-ROD involves the epineurium of the infraorbital nerve with both the endoneurium and perineurium unaffected $[18,19]$. Of note is that no patient in Sogabe et al.'s study and only two patients (33\%) in Lai et al.'s study complained of facial sensory disturbances [2, 3]. Symptomatic trigeminal nerve involvement was less common in cases with positive imaging findings.

Of the cranial nerves affected in patients with IgG4-RD, the bilateral maxillary nerves are rarely involved [17]. A case of an IgG4-related cerebral pseudotumor with perineural spreading along all branches of the trigeminal nerves causing right compressive optic neuropathy has been reported [20]. In the present case, separate involvement of perineural optic nerves without perineural spreading and branches of the trigeminal nerves was reported. Perineural spreading between the maxillary nerve and infraorbital nerve was noted in the present case, but not in previous reports $[17,20]$. No previous IgG4-RON cases mentioned the bilateral maxillary nerves with perineural involvement of infraorbital nerves, which makes the present case exceptional.

The identification of exophthalmos relies not only on accurate measurements but also on normal reference limits. Results from previous studies involving normal healthy subjects from different populations indicate that the normal exophthalmos value for Asians is lower than that of Caucasians. In healthy Asian adults, the upper normal limit of the exophthalmometry measurement is $18.6-19.4 \mathrm{~mm}$, which was lower than the $21 \mathrm{~mm}$ that has traditionally 
been considered as normal for Caucasians $[21,22]$. The distance between the lateral orbital rims in healthy Taiwanese $(105.82 \pm 4.29 \mathrm{~mm})$ was found to be greater than that for Caucasians [22]. In the present case, exophthalmometry measurements were $21.5 \mathrm{~mm}$ with base $112 \mathrm{~mm}$ using the Hertel exophthalmometer.

Thyroid eye disease is the most common cause of unilateral or bilateral proptosis. The extraocular muscle enlargement seen in patients with IgG4-RD could be misinterpreted as being secondary to Graves ophthalmopathy. A distinguishing feature between these two diseases may be the pattern of extraocular muscle involvement. The lateral rectus muscle is the most commonly involved muscle in IgG4$\mathrm{RD}$, while Graves ophthalmopathy tends to spare the lateral rectus muscle until late in the disease course. Furthermore, detections of infraorbital nerve involvement and sinus disease could be typical features of IgG4-RD. Another differential diagnosis is orbital myositis. Orbital myositis most frequently affects the medial rectus muscle, followed by the superior rectus muscle and lateral rectus muscle, and is most often unilateral. In contrast, IgG4-RD favors the lateral rectus muscle and is most commonly bilateral. Unlike IgG4-RD of the orbit, patients with orbital myositis classically present with orbital pain [10].

Optic perineuritis with a doughnut sign and tram-track sign reflects inflamed soft tissues surrounding the optic nerve. Optic meningioma also presents with the tram-track sign, but the lesion is almost unilateral, with the exception of neurofibromatosis type II which may develop bilateral tumors. In the present case, a tram-track sign surrounding the left maxillary nerve and enlarged bilateral infraorbital nerves were also noted. Involvement of the trigeminal nerve, especially the infraorbital nerve, is now a well-recognized manifestation of IgG4-ROD. Thus, the patient reported herein presenting with bilateral doughnut signs of bilateral infraorbital nerves, tram-track sign of the left maxillary nerve, and both doughnut and tram-track signs of bilateral optic nerves could be highly suspected as having IgG4-RD according to the supportive findings of MRI.
This study has a number of limitations. First, the study has a retrospective design; secondly, a standard treatment protocol for IgG4-RON is required to clarify the visual recovery; thirdly, the sample size is relatively small; fourthly, as the optic perineuritis is demonstrated from MRI, it might be misdiagnosed without imaging in cases with subacute presentation. Further studies may need to collect more cases to investigate the risk factors of visual outcomes.

\section{CONCLUSION}

In summary, we presented a clinicopathologic diagnosis of IgG4-RD with bilateral optic perineuritis, enlarged maxillary nerves with perineural spreading to infraorbital nerves, and pansinusitis. Histopathologic examination of biopsy specimen of sinus tissue provided proof of the diagnosis. A poor prognosis is associated with delays in initiating management. Consequently, prompt recognition of IgG4-RON and early treatment with high-dose corticosteroids and an immunosuppressive agent could avert further visual loss.

\section{ACKNOWLEDGEMENTS}

Funding. No funding or sponsorship was received for this study or publication of this article. The Rapid Service Fee was funded by the authors.

Authorship. All named authors meet the International Committee of Medical Journal Editors (ICMJE) criteria for authorship for this article, take responsibility for the integrity of the work as a whole, and have given their approval for this version to be published.

Disclosures. Chih-Heng Hung and Cheng$\mathrm{Yu}$ Lo have no conflicts of interest to disclose.

Compliance with Ethical Guidelines. The patient provided informed consent for his clinical details to be known and signed an informed consent form for publication of the 
case. This study was conducted in compliance with the guidelines of the Declaration of Helsinki.

Data Availability. Data sharing is not applicable for this article as no data sets were generated or analyzed during the current study.

Open Access. This article is licensed under a Creative Commons Attribution-NonCommercial 4.0 International License, which permits any non-commercial use, sharing, adaptation, distribution and reproduction in any medium or format, as long as you give appropriate credit to the original author(s) and the source, provide a link to the Creative Commons licence, and indicate if changes were made. The images or other third party material in this article are included in the article's Creative Commons licence, unless indicated otherwise in a credit line to the material. If material is not included in the article's Creative Commons licence and your intended use is not permitted by statutory regulation or exceeds the permitted use, you will need to obtain permission directly from the copyright holder. To view a copy of this licence, visit http://creativecommons.org/licenses/bync/4.0/.

\section{REFERENCES}

1. Wallace ZS, Deshpande V, Stone JH. Ophthalmic manifestations of IgG4-related disease: Single-center experience and literature review. Semin Arthritis Rheum. 2014;43:806-17.

2. Lai KKH, Chan RYC, Chin JKY, et al. Upper cranial nerve involvement and immunoglobulin G4-related optic neuropathy. Ophthalmology. 2020;127(5):699-703.

3. Sogabe Y, Ohshima K, Azumi A, et al. Location and frequency of lesions in patients with IgG4-related ophthalmic diseases. Graefes Arch Clin Exp Ophthalmol. 2014;252(3):531-8.

4. Umehara H, Okazaki K, Masaki Y. Comprehensive diagnosis criteria for IgG4-related disease (IgG4RD), 2011. Mod Rheumatol. 2012;22:21-30.
5. Goto H, Takahira M, Azumi A. Diagnostic criteria for IgG4-related ophthalmic disease. Jpn J Ophthalmol. 2015;59:1-7.

6. Kashii S. IgG4-related disease: a neuro-ophthalmological perspective. J Neuro-Ophthalmol. 2014;34: 400-7.

7. Lee CS, Harocopos GJ, Kraus CL, et al. IgG4-associated orbital and ocular inflammation. J Ophthalmic Inflamm Infec. 2015;5:15.

8. Lemaitre S, Esquerda GM, Guardiola AC, et al. Colon cancer and IgG4-related disease with orbital inflammation and bilateral optic perineuritis: a case report. Medicine (Baltimore). 2018;97(39):e12197.

9. Kamisawa T, Anjiki H, Egawa N, Kubota N. Allergic manifestations in autoimmune pancreatitis. Eur J Gastroenterol Hepatol. 2009;21(10):1136-9.

10. Tiegs-Heiden CA, Eckel LJ, Hunt $\mathrm{CH}$, et al. Immunoglobulin G4-related disease of the orbit: imaging features in 27 patients. Am J Neuroradiol. 2014;35:1393-7.

11. Abad S, Martin A, Heran F, et al. IgG4-related disease in patients with idiopathic orbital inflammation syndrome: data from the French SIOI prospective cohort. Acta Ophthalmol. 2019;97: e648-e656656.

12. Hickman SJ. Optic perineuritis. Curr Neurol Neurosci Rep. 2016;16:16.

13. Abdel Razek M, Stone JH. Neurologic features of immunoglobulin G4-related disease. Rheum Dis Clin North Am. 2017;43:621-31.

14. Maritati F, Peyronel F, Vaglio A (2020) IgG4-related disease: a clinical perspective. Rheumatology (Oxford). 1;59(Suppl 3):iii123-iii131.

15. Karim AF, Bansie RD, Rombach SM, et al. The treatment outcomes in IgG4-related disease. Neth J Med. 2018;76:275-85.

16. Chwalisz BK, Stone JH. Neuro-ophthalmic complications of IgG4-related disease. Curr Opin Ophthalmol. 2018;29:485-94.

17. Elkhamary SM, Cruz AAV, Zotin MC, et al. Involvement of multiple trigeminal nerve branches in IgG4-related orbital disease. Ophthalmic Plast Reconstr Surg. 2020. https://doi.org/10.1097/IOP. 0000000000001733.

18. Sogabe Y, Miyatani K, Goto R, Ishii G, Ohshima K, Sato $Y$. Pathological findings of infraorbital nerve enlargement in IgG4-related ophthalmic disease. Jpn J Ophthalmol. 2012;56:511-4. 
19. Inoue D, Zen Y, Sato Y, et al. IgG4-related perineural disease. Int J Rheumatol. 2012;2012: 401890.

20. Wu PC, Tien PT, Li YH, et al. IgG4-related cerebral pseudotumor with perineural spreading along branches of the trigeminal nerves causing compressive optic neuropathy: a case report. Medicine (Baltimore). 2017;96(47):e8709.
21. Erb MH, Tran NH, MaCulley TJ, et al. Exophthalmometry measurements in Asians. Invest Ophthalmol Vis Sci. 2003;44:662.

22. Tsai C-C, Kau H-C, Kao S-C, Hsu W-M. Exophthalmos of patients with Graves' disease in Chinese of Taiwan. Eye. 2006;20:569-73. 